associated with the usual 'single spot' method of recording the results. Variable-density and variablearea recording had also been tried and the resulting records, when suitably displayed, could give a striking visual picture of the density variations in the underlying structures without any of the computations normally associated with echo soundings.

The analysis of Earth tide records obtained using Milne-Shaw pendulums was explained by Mr. G. W. Lennon (Liverpool Observatory and Tidal Institute), who enumerated reasons why a digital computer would not be appropriate in this type of work. For example, the removal of spurious diurnal and semidiurnal oscillations would be difficult to programme for a computer, quite apart from the impossibility of producing long records without discontinuities.

Finally, Mr. H. M. Iyer (National Institute of Oceanography) referred to the lack of interest in the study of microseisms. If properly analysed they could yield useful information, and Mr. Iyer described experimental equipment which had recently been developed for the automatic recording of several statistical characteristics of microseisms.

C. M. Munnis

\title{
CHEMISTRY OF CONDENSED TANNINS
}

$\mathrm{T}$ HE Plant Phenolics Group held a meeting on "The Chemistry of Condensed Tannins" at Queen Elizabeth College, University of London, on January 6 under the chairmanship of Prof. R. D. Haworth (University of Sheffield). More than sixty members and guests were present.

In the opening paper on "Acid-catalysed Polymerization of Phenolic Compounds", Dr. B. R. Brown (Oxford) briefly summarized past work on this subject and indicated a possible reaction mechanism. $p$-Methoxybenzyl alcohols condense with phenols preferentially in a position para to a hydroxyl group to yield diphenylmethanes. A suitably substituted flavan, for example 4'-methoxyflavan, undergoes this condensation via ring-fission. Condensation of 4'-methoxyflavan with 7-hydroxyflavan yields a compound containing two flavan units the structure of which is based upon the known para-orientation. of these reactions.

The importance of flavan-3:4-diols in tannin chemistry has led to an investigation of flavan-4-ol, which condenses with phenols, and with 7-hydroxyflavan yields a flavan 'dimer' isolated as the crystalline acetate.

These condensations, like the acid-polymerization of catechin, require high acid concentrations and it is unlikely that they are involved in the conversion of catechins into tannins in plants. This work aims at establishing the structural details of this condensation and at producing 'polymers' of known structure which can be used for the trial of new degradative methods, which may also be valuable for the degradation of condensed tannins.

Dr. D. E. Hathway (British Leather Manufacturers' Research Association), in a paper entitled "Oxidative Polymerization Mechanisms for Catechins", first briefly reviewed the chemistry of the oxidation of $o$ - and $p$-dihydroxy- and vic-trihydroxyphenols, which he illustrated by reference to the synthesis of the mould metabolite atromentin, to the formation of purpurogallin and the synthetic melanins, and to the transformation of gallates into ellagates. Possible mechanisms for the oxidation of coniferyl alcohol by laccase, and their relevance to lignin and lignan formation were also discussed.

Studies on the oxidation of $(+)$-catechin in aqueous and non-aqueous media and on the autoxidation of flavans related to catechin confirm that autoxidation of catechin to a polymer (catechin-tannin) proceeds via the corresponding $o$-quinone followed by intermolecular $\mathrm{C}-\mathrm{C}$ linkage. The evidence suggests that the most likely linkage is between the 8-carbon of one molecule and the 6 -carbon of the second. The isolation, albeit in small yield, of $m$-hemipinic acid by oxidative degradation of the methylated reduced polymer substantiates this supposition. Further confirmation for the presence of head-to-tail units in the polymer was obtained from a study of the autoxidation and the enzymic oxidation of mixed substrates which resemble the head and tail of $(+)$-catechin. Enzymic oxidation by mushroom, potato and tobacco polyphenoloxidases proceeds at a faster rate and lower temperature than the autoxidation of catechin, and gives a product precisely similar to the autoxidation polymer.

The heartwood of Acacia catechu and the leaves of Uncaria gambir contain considerable quantities of (-)-epicatechin and (+)-catechin, respectively. The isolation, therefore, of purified tanning fractions in good yield from these sources, with properties similar to the autoxidation and enzymic oxidation polymers of catechin, strongly suggests that these tannins are formed from epicatechin and catechin, respectively, by the above mechanism.

Studies on the formation of oak-bark tannin indicate that $(+)$-catechin, (+)-gallocatechin and leucodelphinidin, which are formed in the leaves, are translocated via the sieve-tube system of the phloem to the cambium, where preferential enzymic oxidation of the pyrogallol phenols occurs. The resulting tail-to-tail polymer is stored in the bark. The remaining tannin action of the bark can be attributed to the occurrence of complex leucoanthocyanins.

In a paper on "Oxidative Condensation in Tea Fermentation", Dr. E. A. H. Roberts (Indian Tea Association) said that, according to Hathway and Seakins, oxidation of $(+)$-catechin is followed by a condensation between the catechol-o-quinone group of one molecule and the phloroglucinol ring of another. If phloroglucinol is added to an enzymocatechol system, different intermediate- and endproducts are formed, indicating a condensation of o-benzoquinone with highly active phloroglucinol. Oxidation of pyrogallol to purpurogallin, however, is not affected by the presence of phloroglucinol, which illustrates the very high reactivity associated with the hydroxy-o-benzoquinone system. Enzymic oxidations of (-)-epigallocatechin and its gallate are similarly unaffected by the addition of phloroglucinol, but the addition of pyrogallol to these systems yields derivatives of purpurogallin (flavanotropolones). These observations suggest that, following oxidation 
of the gallocatechins, a condensation takes place between the pyrogallol groups of two separate molecules.

Gallic acid and pyrogallol will not condense to form purpurogallincarboxylic acid unless both substances are oxidized, and this suggests that in the gallocatechin series tho condensation takes place between two oxidized molecules. This is supported by the results of a study of the conditions required for the formation of purpurogallin.

The oxidations of (-)-epigallocatechin and its gallate are followed by a linkage of two molecules of the resultant $o$-quinones to form the $o$-quinone of a bisflavanol with the two pyrogallol groups joined by a $\mathrm{C}-\mathrm{C}$ linkage. Further condensations of this type are excluded on steric grounds, and it is suggested that the primary eondensation products undergo either oxidation or reduction. Reduction leads to the formation of one of three possible bisflavanols, whereas oxidation, if a galloyl group is present, could lead to the formation of one of two purpurogallin derivatives. The available evidence supports the view that the theaflavins of black tea have these structures.

Prof. K. Freudenberg (Heidelberg) said that the term leucoanthocyani(di)n was a misnomer for those naturally occuring substances which on treatment with hot mineral acid either under aerobic or anaerobic conditions yield anthocyanidins. He proposed that they be named either 'pre- or pro-anthocyanins'.

Dr. T. Swain (Low Temperature Research Station, Cambridge), in introducing his paper on "Methods for the Investigation of Complex Leucoanthocyanins", also referred to the confusion in the nomenclature of leucoanthocyanins. $\mathrm{He}$ suggested that leucoanthoeyanins of known structure be called by their systematic name, whereas polymers and co-polymers of hydroxyflavans ('flavols') be known as 'flavolans'. Those flavolans which yielded anthocyanidins on treatment with hot mineral acid would be known as 'flavylogens'. Dr. Swain then described methods for separating polymeric leucoanthocyanins into size groups and for analysis of their end groups.

Mr. W. E. Hillis (Division of Forest Products, Commonwealth Scientific and Industrial Research Organization, Australia), in his paper on the formation of condensed tannins in plants (read by Dr. E. C. Bate-Smith), recalled the probable role of shikimic acid and related alicyclic acids in the biosynthesis of polyphenols. Shikimic acid is present in appreciable quantities in the actively proliferating cambium of eucalypts. A linear relation exists between the amounts of shikimic acid and the weights of enlarging leaves of young Eucalyptus sieberiana trees, but this relationship does not hold for the fully-grown leaf; later the amount of shikimic acid in mature leaves diminishes with age. The final stages of the biosynthesis of shikimic acid can occur in the dark, and therefore shikimic acid present elsewhere in the tree need not necessarily originate in the leaves. The distribution of shikimic acid in leaves differs from that of the soluble polyphenols.

Comparative studies of the polyphenols in the cambium, sapwood and heartwood of three Eucalyptus species revealed the samo general pattern of polyphenols, but shikimic acid was absent from the heartwood and only traces occurred in the developing sapwood. Catechin was less in the heartwood, whereas ellagic and gallic acids increased. On the basis of these facts a possible scheme for the biosynthesis of the heartwood polyphenols was outlined.

Mr. Hillis recalled that he had previously suggested (Society of Leather Trades Chemists Symposium on Vegetable Tannins, 1956, p. 127) that as certain tannins contain appreciable amounts of polymeric leucoanthocyanins, monomeric forms, originating in the leaves, could be the precursors of these substances. Present information, however, favours the formation of heartwood leucoanthocyanins in situ. Adaptation of the leucoanthocyanin reaction has enabled the distribution of leucoanthocyanins in plant tissues to be studied by moans of coloured photomicrographs.

In dealing with flavans of the resorcinol series in relation to the chemistry of commercially important condensed tannins, Dr. D. G. Roux (Leather Industries Research Institute, Grahamstown, South Africa) said that an examination of previous work on wattle and quebracho extracts indicated that no direct evidence had been provided for the occurrence of catechins or flavan-3:4-diols as basic units in the corresponding tannins. The stereochemistry of the recently isolated black wattle catechin ( - ). $3: 7: 3^{\prime}: 4^{\prime}: 5^{\prime}$-pentahydroxyflavan and (- )-7:3': $4^{\prime}$-trihydroxyflavan-3:4-diol of quebracho heartwood was discussed. The progress of condensation of these compounds in the wood was examined by anthoeyanidin formation, by number average molecular weights and by tannin analyses on radial and vertical drillings. These methods afford possible biochemical evidence of the presence of leucoanthocyanidins as intact flavan-3:4-diol units in these condensed tannins. The mode of cross-linkage in these condensed tannins must allow for its labile nature.

The chairman encouraged a vigorous discussion at the end of each paper, and among those participating were Drs. White and King (Forestal Central Laboratories), Bate-Smith, Thompson and Whalley. J. W. T. SEakins

\section{BAMBOOS AND NUTMEGS}

$\mathrm{T}$ WO important taxonomic studies have recently been published in The Gardens' Bulletin (Singapore, 16, 1958), namely, "Bamboos of Malaya" by R. E. Holttum, and "A Revision of the Malayan Myristicaceae" by J. Sinclair.

Holttum has pointed out that the classification of the bamboos, as of other Gramineae, is based on inflorescence, flower and fruit characters; and in so far as such work tends to be done in herbaria, remote from the tropical collecting grounds, much information that would be of great use to the field botanist fails to be included. In fact, what the field botanist mostly sees are bamboos not in flower. Hence the value of vegetative characters, in particular the culm-sheaths, in field observations. This, indeed, is the kind of information possessed by the Malays, by whom the many kinds of bamboo are put to manifold domestic and other uses. Accordingly, in the present very full and informative work, classifications based on both vegetative and reproductive characters are given, 